\title{
Lack of association between rare mutations of the SIAE gene and rheumatoid arthritis in a Han Chinese population
}

\author{
D.D. Zhang ${ }^{1,2 *}$, F. He ${ }^{3 *}$, H.T. Liü* ${ }^{4 *}$ F. Hao ${ }^{1}$ and J. Zhu ${ }^{5}$ \\ ${ }^{1}$ Sichuan Key Laboratory for Disease Gene Study, \\ Sichuan Academy of Medical Sciences and Sichuan Provincial People's Hospital, \\ Sichuan, China \\ ${ }^{2}$ Department of Health Management, \\ Sichuan Academy of Medical Sciences and Sichuan Provincial People's Hospital, \\ Sichuan, China \\ ${ }^{3}$ School of Life Science and Biotechnology, \\ Mianyang Normal University, Sichuan, China \\ ${ }^{4}$ Guangya School, Guangdong, China \\ ${ }^{5}$ Department of Rheumatology and Immunology, \\ Sichuan Academy of Medical Sciences and Sichuan Provincial People's Hospital, \\ Chengdu, Sichuan, China \\ *These authors contributed equally to the study. \\ Corresponding author: D.D. Zhang \\ E-mail: zhangdd25@126.com
}

Genet. Mol. Res. 14 (4): 14162-14168 (2015)

Received July 16, 2015

Accepted September 29, 2015

Published October 29, 2015

DOI http://dx.doi.org/10.4238/2015.October.29.38

ABSTRACT. The function of rare genotypes encoding defective variants of sialic acid acetylesterase (SIAE) in some autoimmune diseases, including rheumatoid arthritis (RA), is ambiguous. We determined whether mutations in the SIAE gene are responsible for RA in a Han Chinese population. DNA was prepared from the venous leukocytes of 444 RA patients and 647 normal controls. The coding regions and adjacent intron sequences of SIAE were amplified by polymerase chain reaction. The products were 
then subjected to sequencing analysis. The detected variations were further evaluated in the normal controls and available family members by sequencing. Seven variants of RA were identified in this study, including four known single nucleotide polymorphisms SNPs (rs7941327, rs7941523, rs 1942663, and rs12282107) and three novel SNPs. The genomic positions of the three novel SNPs are chr11:124013712, chr11:124023268, and chr11:124044505. No significant differences in the seven SNPS of SIAE were observed between patients with RA and controls in this cohort $(P>$ 0.05). Three novel variations and four known SNPs in SIAE were detected in the Chinese RA patients and normal controls. Our results imply that SIAE does not play a major role in RA in this population.

Key words: Rheumatoid arthritis; Sialic acid acetylesterase; Sequencing; Single nucleotide polymorphism; Han Chinese population

\section{INTRODUCTION}

Rheumatoid arthritis (RA) is considered a systemic autoimmune disease. It causes chronic inflammation of the joints and may affect many different tissues and organs. The prevalence of RA is roughly $0.2-0.3 \%$ in China and nearly $0.8 \%$ in the Western world (Silman and Hochberg, 2001). Although the cause of RA has not been fully elucidated, it is believed that the combined effect of genetic and environmental factors, as well as exposure to certain infections, may cause the errant activation of the immune system in susceptible individuals. Several studies have shown that a group of genes are implicated in susceptibility to RA and its severity, including class II major histocompatibility complex genes, PTPN22 (protein tyrosine phosphatase, non-receptor type 22), and PADI4 (peptidyl arginine deiminase) (Chung and Criswell, 2007; Lee et al., 2007; Taneja and David, 2010).

The sialic acid acetylesterase (SIAE) gene encodes an enzyme that removes 9-O-acetylation modifications from sialic acid. It negatively regulates $B$ lymphocyte antigen receptor signaling and is required for the maintenance of immunological tolerance in mice. Compelling evidence suggests that SIAE may play a critical role in the pathogenesis of autoimmune diseases (Cariappa et al., 2009; Pillai et al., 2009).

Recently, studies on SIAE in European populations have attracted particular interest owing to the identification of rare loss-of-function genetic variants in the SIAE gene that appear to predispose individuals to multiple autoimmune diseases. Surolia et al. (2010) identified distinct nonsynonymous SIAE risk variant genotypes of autoimmune diseases in individuals of European origin. Hirschfield et al.(2012) revealed the potential role of functionally defective SIAE variants in the risk of primary biliary cirrhosis (PBC). These studies showed that defective SIAE variants have a strong effect on susceptibility to autoimmunity. Furthermore, studies by other investigators have confirmed the role of SLAE mutation in the development of other chronic diseases such as diabetes. Szymański et al. (2011) tested the homozygous Met89Val substitution of SIAE in patients with Graves' disease (GD) and type 1 diabetes mellitus (T1DM) patients in a Polish population. Hunt et al. (2011) replicated and extended the SIAE findings of Surolia et al. (2010) in a much larger independent study of autoimmune and chronic immune diseases. However, Hunt et al.'s results illustrated that rare and functional SIAE variants are not associated with autoimmune disease risk. In the present study, we sought to determine whether the variations of SIAE are associated with RA in a Han Chinese population. 


\section{MATERIAL AND METHODS}

\section{Study population}

In total, 444 patients with RA and 647 normal age-matched controls were recruited. All the patients enrolled in this study had received a clinical diagnosis of RA at least two years before, and the mean duration of the disease was five years. The normal age-matched controls and the RA patients, including individuals who had normal joints upon examination, were recruited at the Rheumatology and Immunology Clinic of the Sichuan Provincial People's Hospital and North Sichuan Medical College, China. All the participants underwent a standard examination protocol according to the guidelines of the American Rheumatism Association, as noted previously: (http://medcalc3000.com/RheumatoidArthritis.htm). In the normal controls, no sign of joint or other autoimmune diseases were detected. Clinical information about the cases and controls is provided in Table 1. All the participants signed an informed consent form.

Table 1. Characteristics of rheumatoid arthritis (RA) cases and controls matched for age and ethnicity.
\begin{tabular}{lcccccc}
\hline Subject & Total number & anti-CCP-positive & RF-positive & Male & Female & Average age \\
\hline Cases & 444 & 297 & 411 & 176 & 268 & $62 \pm 6.3$ \\
Controls & 647 & 0 & 0 & 312 & 335 & $60 \pm 5.8$ \\
\hline
\end{tabular}

$\mathrm{CCP}=$ cyclic citrullinated peptide; $\mathrm{RF}=$ rheumatoid factor; \pm represents standard deviation.

\section{Mutation analysis}

Genomic DNA was prepared from peripheral leukocytes as described previously (Hunt et al., 2011). Table 2 lists the primers used to amplify the coding exons and adjacent introns of SIAE (NCBI human genome build 36.3, NC_000011.9 for genomic DNA, NM_032811.2 for mRNA, and NP_001186851.1 for proteins). The nucleotide sequences of the SIAE exons were determined using an Applied Biosystems 3710 DNA Analyzer (Carlsbad, CA, USA). The sequencing results from the cases and the controls, as well as the SIAE consensus sequences, were compared to identify variations. The variations were confirmed by bidirectional sequencing.

\section{Statistical analysis}

The $\mathrm{X}^{2}$ test was used to analyze the Hardy-Weinberg equilibrium (HWE) of the SNPs. Allele and genotype frequencies between the cases and the controls were compared by $X^{2}$ analysis. Statistical significance was defined as $P<0.05$. All statistical analyses were performed using SPSS version 10.0 (SPSS Inc., Chicago, IL, USA).

\section{RESULTS}

Among the 444 patients with RA, 297 were anti-cyclic citrullinated peptide (CCP) antibodypositive, while 411 were rheumatoid factor (RF)-positive. The frequency of females was 268/444 $(60.4 \%)$. The patients diagnosed with RA had an average age of 62 years (range 55.7-68.3 years), and the average age of the normal controls was 60 years (range 54.2-65.8 years) (Table 1). 
Table 2. Primers used for the amplification and sequencing of sialic acid acetylesterase gene (SIAE).

\begin{tabular}{|c|c|c|c|c|}
\hline Exon number & Direction & Primer sequence & Size of product (bp) & Annealing temperature $\left({ }^{\circ} \mathrm{C}\right)$ \\
\hline \multirow[t]{2}{*}{1} & $\mathrm{~F}$ & 5'-AAGACCCTTCTGACCTAA-3' & 351 & 52 \\
\hline & $\mathrm{R}$ & 5'-TAAAATCTGAAGGACCCA-3' & & \\
\hline \multirow[t]{2}{*}{2} & $\mathrm{~F}$ & 5'-AGCTGACTAGCCACTGATCACAGA-3' & 460 & 58 \\
\hline & $\mathrm{R}$ & 5'-GCTCAGCCCCCAACCAACCA-3' & & \\
\hline \multirow[t]{2}{*}{3} & $\mathrm{~F}$ & 5'-CCAGTGAAAAGACTCCAGTGTGGG-3' & 254 & 58 \\
\hline & $\mathrm{R}$ & 5'-TGCAGCACACCCCAGCATACG-3' & & \\
\hline \multirow[t]{2}{*}{4} & $\mathrm{~F}$ & 5'-TTGCTGATGCCCCACTGGCTG-3' & 374 & 60 \\
\hline & $\mathrm{R}$ & 5'-GCACATCCCCACACCAATGCCC-3' & & \\
\hline \multirow[t]{2}{*}{5} & $\mathrm{~F}$ & 5'-CATAGAAAGCTGACCGATAC-3' & 471 & 55 \\
\hline & $\mathrm{R}$ & 5'-АСТАСCCTGCTCACCCAC-3' & & \\
\hline \multirow[t]{2}{*}{6} & $\mathrm{~F}$ & 5'-CCCCACAGAGGACGATGACCCT-3' & 338 & 60 \\
\hline & $\mathrm{R}$ & 5'-CCCCACAGAGGACGATGACCCT-3' & & \\
\hline \multirow[t]{2}{*}{7} & $\mathrm{~F}$ & 5'-CAGTGCAGCCCATTGGTTTTGGT-3' & 337 & 58 \\
\hline & $\mathrm{R}$ & 5'-TCCACTTAGGGCGCTAACCCA-3' & & \\
\hline \multirow[t]{2}{*}{8} & $\mathrm{~F}$ & 5'-ACAGAGGCTGGGAATAGCCACA-3' & 361 & 58 \\
\hline & $\mathrm{R}$ & 5'-GCCCTGCTTCTCCTTGTTGCCC-3' & & \\
\hline \multirow[t]{2}{*}{9} & $\mathrm{~F}$ & 5'-СTCTCCGATGATGCTTCCCTCAGT-3' & 234 & 58 \\
\hline & $\mathrm{R}$ & 5'-AGCAGGTGGCAGAAGAGGGCAA-3' & & \\
\hline \multirow[t]{2}{*}{10} & $\mathrm{~F}$ & 5'-CCCTGCCGGGAACCCACTTTC-3' & 179 & 60 \\
\hline & $\mathrm{R}$ & 5'-GGTCTCGGAGCCCGCACAGG-3' & & \\
\hline
\end{tabular}

$\mathrm{F}=$ forward; $\mathrm{R}=$ reverse.

Complete sequencing of the coding regions and the adjacent intron regions of SIAE in 1091 DNA samples identified three novel mutations and four known SNPs (Figure 1). The results of this analysis revealed two synonymous variations (c.1452C $>$ T and c.736G $>A$ ) and five missense variations (Table 3). Among the five missense variations, the c.1400G>A mutation (p.A467V), a known polymorphism, was detected in 11 of the 444 patients with RA (10 heterozygous and 1 homozygous), as well as in 12 of the 647 controls (12 heterozygous). The c.1316C>T mutation, a novel heterozygous variation (p.G419E) named novel SNP1, was detected in 9 of the 444 patients with RA (9 heterozygous), as well as in 23 of the 647 controls ( 1 heterozygous and 24 homozygous). The c.1256G>A mutation, another novel heterozygous variation (p.D246N) named novel SNP2, was detected in 15 of the 444 patients with RA (1 heterozygous and 14 homozygous), as well as in 15 of the 647 controls (15 heterozygous). The c.212T>C mutation (p.K71A), a known polymorphism, was detected in 86 of the 444 patients with RA (78 heterozygous and 8 homozygous), as well as in 148 of the 647 controls (138 heterozygous and 10 homozygous). The c.190C>T mutation (p.G64S), a novel polymorphism named novel SNP3, was detected in 93 of the 444 patients with RA ( 89 heterozygous and 4 homozygous) as well as in 119 of the 647 controls (112 heterozygous and 7 homozygous).

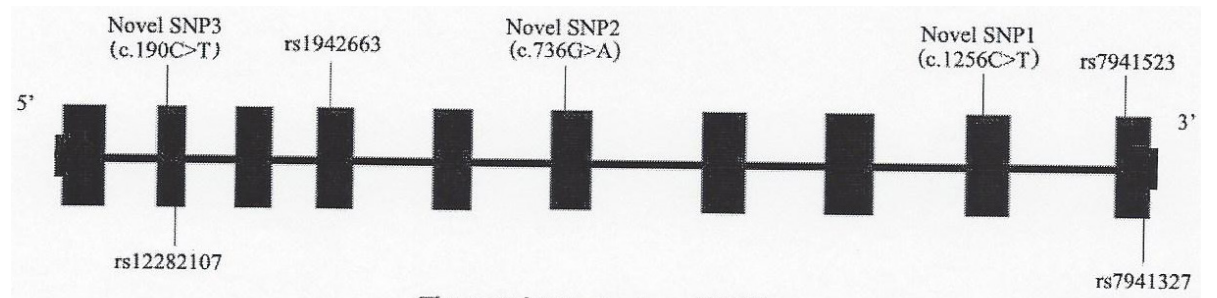

The exon-intron structure of SIAE

Figure 1. Schematic diagram of the exon-intron structures and variants identified in the study of the sialic acid acetylesterase gene (SIAE). Black boxes represent exons. The location and ID of single-nucleotide polymorphisms (SNPs) genotyped in the SIAE panel are shown. 
Table 3. Sialic acid acetylesterase gene $(S I A E) v$ ariants detected in rheumatoid arthritis patients and healthy controls in a Han Chinese population by direct sequencing of all the exons.

\begin{tabular}{|c|c|c|c|c|c|c|c|c|}
\hline \multirow[t]{2}{*}{ SNP ID } & \multirow[t]{2}{*}{ Exon number } & \multirow[t]{2}{*}{ Genomic position } & \multirow[t]{2}{*}{ Allele* $^{*}$} & \multirow[t]{2}{*}{ Residue change } & \multicolumn{2}{|c|}{ Genotype counts $^{\dagger}$} & \multirow[t]{2}{*}{$P$ value } & \multirow[t]{2}{*}{ OR $(95 \% \mathrm{Cl})$} \\
\hline & & & & & Cases & Controls & & \\
\hline rs7941327 & 1 & chr11:124012177 & $\mathrm{T} / \mathrm{C}$ & T484T & $9 / 90 / 345$ & $10 / 131 / 506$ & 0.731 & $1.05(0.81-1.36)$ \\
\hline rs7941523 & 1 & chr11:124012229 & $\mathrm{A} / \mathrm{G}$ & A467V & $1 / 10 / 433$ & $0 / 12 / 635$ & 0.368 & $1.46(0.65-3.27)$ \\
\hline Novel SNP1 & 2 & chr11:124013712 & $\mathrm{T} / \mathrm{C}$ & G419E & $0 / 9 / 434$ & $1 / 24 / 622$ & 0.076 & $0.50(0.23-1.07)$ \\
\hline Novel SNP2 & 5 & chr11:124023268 & $A / G$ & D246N & $1 / 14 / 430$ & $0 / 15 / 632$ & 0.227 & $1.56(0.77-3.17)$ \\
\hline rs1942663 & 7 & chr11:124029837 & $\mathrm{G} / \mathrm{A}$ & S156S & $5 / 82 / 357$ & $8 / 127 / 512$ & 0.610 & $0.19(0.12-0.30)$ \\
\hline rs12282107 & 9 & chr11:124035880 & $\mathrm{C} / \mathrm{T}$ & D87Y & 8/78/358 & $10 / 138 / 499$ & 0.251 & $0.17(0.10-0.27)$ \\
\hline Novel SNP3 & 9 & chr11:124044505 & $\mathrm{T} / \mathrm{C}$ & G64S & $4 / 89 / 351$ & $7 / 112 / 528$ & 0.378 & $0.21(0.13-0.35)$ \\
\hline
\end{tabular}

$\mathrm{SNP}=$ single-nucleotide polymorphism. ${ }^{*}$ Minor allele/major allele. ${ }^{~}$ The genotype counts are presented as homozygote/ heterozygote/wild type.

There was no significant association $(P>0.05)$ between the RA patients and the normal controls in the Chinese population with any of the SIAE variations.

\section{DISCUSSION}

SIAE plays an important role in the maintenance of B-cell tolerance. It influences B-cell signaling and development, and participates in inhibitory signaling mechanisms (Pillai, 2013; Yamamoto et al., 2014). SIAE represses B-cell receptor (BCR) signaling via the SIAE-Siglec-LynSHP-1 pathway. Siglec-2 is a member of the Siglec family that binds to $\mathrm{N}$-glycans containing sialic acid via an $\alpha-2,6$ linkage to an $\mathrm{N}$-acetyllactosamine moiety of the BCR (Siglec is an abbreviation for sialic acid-binding immunoglobulin-type lectin). Siglec- 2 is rapidly phosphorylated on its cytoplasmic inhibitory tyrosines by Lyn, an Src family tyrosine kinase. Following the phosphorylation of Siglec-2, Src homology region 2 domain-containing phosphatase-1 (SHP-1) is recruited to the cytoplasmic tail of CD22, thereby inhibiting BCR signaling. SIAE can also remove O-acetyl moieties from 9-O-acetylated sialic acid (Stoddart at al., 1996; Takematsu et al., 1999). Thus, this pathway impairs BCR signaling. However, mutations in SIAE presumably lead to autoimmunity by enhancing the signal strength of BCR.

Recently, Surolia et al. (2010) investigated SIAE risk variant genotypes in 923 European patients with 10 common autoimmune diseases and 648 controls. All the heterozygous loss-offunction SIAE mutations showed a dominant negative function; whereas a homozygous secretiondefective polymorphic variant of SIAE was catalytically active and lacked the dominant negative function. This was seen in eight autoimmune subjects but not in any of the control subjects. Therefore, the researchers concluded that rare and polymorphic variants of SIAE give rise to defects in function, which points to an association between SIAE and autoimmune disorders. Hirschfield et al. (2012) sequenced the SIAE gene in 1450 PBC cases and 2957 healthy controls, and identified six cases with functional nonsynonymous SIAE mutations and functional SIAE variants that were associated with PBC risk.

However, there is still some controversy regarding the association of SIAE with autoimmune diseases. A study by Szymański et al. (2011) comprising 561 GD patients, 379 T1DM patients, and 1822 controls found that the prevalence of Met89Val homozygosity was similar among patients and controls, yielding ORs of 0.93 and 0.69 for GD and T1DM, respectively. This study highlighted the fact that the strong effect of defective SIAE variants on susceptibility to autoimmunity needs to be investigated further. Moreover, Hunt et al. (2011) tested for the presence of the SIAE variant 
in 66,924 samples from five ethnic groups with autoimmune diseases and found homozygotes of the variant in all cases and controls. The variant encoding SIAE p.Met89Val was genotyped in 4805 offspring-parent trios of European origin in five autoimmune diseases. The study found no evidence indicating an effect of the p.Met89Val alteration on autoimmune disease risk. An analysis of eight additional rare SIAE variants in 43,378 subjects identified no functional SIAE variants as potential factors for autoimmune disease risk (Hunt et al.,2011). Finally, Gan et al. (2012) analyzed nine SIAE gene variants in a UK cohort of 378 subjects with autoimmune Addison's disease (AAD) and 387 healthy controls. Their analysis did not support a role for rare variants in SIAE in the pathogenesis of AAD. Thus, further studies are needed to validate the relationship between the SIAE gene and autoimmune diseases.

In this study, we resequenced the 10 exons of SIAE in the 444 RA patients and the 647 normal controls of Han origin. We then attempted to identify novel, rare, and functional mutations in SIAE and investigate the association between these SNPs in SIAE and RA. We detected seven SNPs, including three novel SNPs and four known SNPs. Among them, two variations cause synonymous-coding, while five were missense. None of the seven SNPs showed a significant association with RA $(P>0.05)$ (Table 3$)$. Consistent with the results obtained in Dutch populations (Hunt et al., 2011), our study did not identify SIAE as a significant risk factor for RA in a Han Chinese population. This suggests that possible heterogeneity among different ethnicities or genetic variants in the SIAE gene is not associated with RA.

In summary, we established a replication cohort of 444 RA patients and 647 controls to determine the potential presence of SIAE variants in a Han Chinese population. Our results suggest that SIAE is unlikely to play a major role in RA. Additional studies will be necessary to clarify these results.

\section{Conflicts of interest}

The authors declare no conflict of interest.

\section{ACKNOWLEDGMENTS}

The authors thank all the patients and family members for their participation. This study was supported by grants from the Scientific Research Project of Sichuan Provincial Health Department (\#110140 to F. He and \#100447 to J. Zhu), the Scientific Research Project of Sichuan Provincial Education Department (\#13zb0122 to F. He), and the Department of Science and Technology of Sichuan Province, China (\#06SG1964 to D.D. Zhang).

\section{REFERENCES}

Cariappa A, Takematsu H, Liu H, Diaz S, et al. (2009). B cell antigen receptor signal strength and peripheral B cell development are regulated by a 9-O-acetyl sialic acid esterase. J. Exp. Med. 206: 125-138.

Chung SA and Criswell LA (2007). PTPN22: its role in SLE and autoimmunity. Autoimmunity 40: 582-590.

Gan EH, MacArthur K, Mitchell AL and Pearce SH (2012). The role of functionally defective rare germline variants of sialic acid acetylesterase in autoimmune Addison's disease. Eur. J. Endocrinol. 167: 825-828.

Hirschfield GM, Xie G, Lu E, Sun Y, et al. (2012). Association of primary biliary cirrhosis with variants in the CLEC16A, SOCS1, SPIB and SIAE immunomodulatory genes. Genes Immun. 13: 328-335.

Hunt KA, Smyth DJ, Balschun T, Ban M, et al. (2011). Rare and functional SIAE variants are not associated with autoimmune disease risk in up to 66,924 individuals of European ancestry. Nat. Genet. 44: 3-5. 
Lee YH, Rho YH, Choi SJ, Ji JD, et al. (2007). PADI4 polymorphisms and rheumatoid arthritis susceptibility: a meta-analysis. Rheumatol. Int. 27: 827-833.

Pillai S (2013). Rethinking mechanisms of autoimmune pathogenesis. J. Autoimmun. 45:97-103.

Pillai S, Cariappa A and Pirnie SP (2009). Esterases and autoimmunity: the sialic acid acetylesterase pathway and the regulation of peripheral B cell tolerance. Trends Immunol. 30: 488-493.

Silman A and Hochberg MC (2001). Epidemiology of the rheumatic diseases. 2nd edn. Oxford University Press, New York.

Stoddart A, Zhang $Y$ and Paige CJ (1996). Molecular cloning of the CDNA encoding a murine sialic acid-specific 9-O-acetylesterase and RNA expression in cells of hematopoietic and non-hematopoietic origin. Nucleic Acids Res. 24: 4003-4008

Surolia I, Pirnie SP, Chellappa V, Taylor KN, et al. (2010). Functionally defective germline variants of sialic acid acetylesterase in autoimmunity. Nature 466: 243-247.

Szymański K, Skórka A, Szypowska A, Bednarczuk T, et al. (2011). Functionally defective germline variant of sialic acid acetylesterase (Met89Val) is not associated with type 1 diabetes mellitus and Graves' disease in a Polish population. Tissue Antigens 78: 214-216.

Takematsu H, Diaz S, Stoddart A, Zhang Y, et al. (1999). Lysosomal and cytosolic sialic acid 9-O-acetylesterase activities can Be encoded by one gene via differential usage of a signal peptide-encoding exon at the $\mathrm{N}$ terminus. J. Biol. Chem. 274: 25623-25631.

Taneja V and David CS (2010). Role of HLA class II genes in susceptibility/resistance to inflammatory arthritis: studies with humanized mice. Immunol. Rev. 233: 62-78.

Yamamoto M, Iguchi G, Bando H, Fukuoka H, et al. (2014). A missense single-nucleotide polymorphism in the sialic acid acetylesterase (SIAE) gene is associated with anti-PIT-1 antibody syndrome. Endocr. J. 61: 641-644. 